\title{
Posterior Cruciate Ligament Reconstruction and Posterolateral Corner Injury
}

\author{
Young Mo Kim, MD \\ Department of Orthopedic Surgery, Chungnam National University School of Medicine, Daejeon, Korea
}

Posterior cruciate ligament (PCL) injuries comprise 3\% to $38 \%$ of all acute knee injuries ${ }^{1)}$, and various operative methods for posterior cruciate ligament reconstruction (PCLR) have been developed over the past several years. However, controversy regarding surgical techniques for PCLR still remains.

Posterolateral corner (PLC) injuries are rare and frequently encountered combined with anterior cruciate ligament (ACL) or PCL tears. It is often difficult to make an accurate diagnosis immediately after an acute injury, and a PLC injury can be overlooked or misdiagnosed. Various operative treatment methods for PLC injuries have been reported depending on the degree of injury and the timing of surgery. Considering that the incidence of PLC injuries has risen owing to the increase in motor vehicle accidents and athletic traumas, accurate in-depth understanding of PLC injuries is even more important.

The current issue of Knee Surgery \& Related Research contains three articles on PCLR and PLC injuries including one metaanalysis of single-bundle (SB) versus double-bundle (DB) PCLR, one case report describing arthroscopic operative treatment of an avulsed PCL injury from the tibia, and one review article on the current concept of PLC injuries of the knee.

Previously, the anterolateral bundle (ALB) of PCL was considered to be more associated with linear stiffness and ultimate loading than the posteromedial bundle $e^{2,3)}$. Thus, the focus was

Correspondence to: Young Mo Kim, MD

Department of Orthopedic Surgery, Chungnam National University School of Medicine, 282 Munhwa-ro, Jung-gu, Daejeon 35015, Korea Tel: +82-42-338-2482, Fax: +82-42-338-2480

E-mail: osdr69@cnu.ac.kr

This is an Open Access article distributed under the terms of the Creative Commons Attribution Non-Commercial License (http://creativecommons.org/licenses/by-nc/4.0/) which permits unrestricted non-commercial use, distribution, and reproduction in any medium, provided the original work is properly cited. mainly on reconstruction of the ALB of PCL. However, early studies showed its limited efficacy, such as persistent postoperative instability, particularly residual posterior laxity in full extension $^{4)}$. Therefore, some authors have suggested that DB PCLR is superior to SB PCLR in terms of restoration of posterior laxity of the knee ${ }^{5)}$. Also, many authors reported that DB PCLR would be more beneficial in restoring the intact knee function ${ }^{6-8)}$. However, such studies were confined to in vitro studies, and there are no clinical studies demonstrating that DB PCLR is superior to SB PCLR. In addition, DB PCLR is not recommended as the preferred surgical procedure because of the longer operation time, technical difficulty, and larger trauma. Thus, based on clinical trials, the superiority between the two techniques in terms of clinical improvement remains inconclusive.

The meta-analysis included in this issue of Knee Surgery \& Related Research reviewed randomized controlled trials (RCTs) on the outcomes of SB and DB PCLR. Authors concluded that both techniques for PCLR were useful for restoration of knee stability and improved knee function. However, which technique yields better improvement in clinical outcomes remains unclear. Therefore, to verify and further corroborate the clinical results of SB and DB PCLR, more larger-scale, high-quality RCTs are encouraged.

In the case report of a tibial avulsion fracture of the PCL, the authors performed arthroscopic pullout re-approximation using three usual portals (anteromedial, anterolateral and posteromedial portals) for the PCL injury.

PLC injury is rare and frequently a part of multiple ligament injury of the knee; thus, it can be overlooked or misdiagnosed initially. If left untreated, it can be the cause of chronic pain, residual posterolateral instability, and failure of the reconstructed ACL or PCL graft. Therefore, early detection and proper treatment of injured PLC structures are of utmost importance. The review 


\section{Kim. Posterior Cruciate Ligament Reconstruction and Posterolateral Corner Injury}

article published in the current issue summarizes the recent literatures regarding the anatomy and biomechanics of PLC and diagnosis, classification, surgical treatments, and postoperative complications of PLC injury.

Isolated grade I and II PLC injuries can be treated non-operatively. Although there is a lack of reports on the outcome of non-operative treatment, minimal radiographic changes were reported in an 8-year follow-up study of patients with an early mobilization protocol ${ }^{9,10)}$. For isolated grade III PLC injuries and grade II PLC injuries combined by other structural injuries, operative treatment is recommended. The interval between the time of injury and operation is a major factor in the selection of an operative method. Usually, an acute injury is defined as an injury that occurred within 3 weeks prior to treatment and can be treated with direct repair or augmentation although recent studies have reported that primary repair is not sufficient to treat injuries of the lateral collateral ligament, popliteus tendon, or popliteofibular ligament ${ }^{11,12)}$. If the grade of injury is severe or tissue is not vital enough, augmentation or reconstruction can be considered instead of primary repair. Outcomes of repair of acute PLC injuries are better when performed early in the acute stage ${ }^{13)}$. If the surgery is performed within the first 2 weeks of injury, the anatomy is much easier to identify and anatomic repair can often be achieved with ease ${ }^{14)}$.

A chronic injury of the posterolateral structure is the one that has persisted for more than 3 weeks following injury. The torn structure becomes fibrotic scar tissue and direct repair can be difficult owing to tissue adhesion. Moreover, joint stiffness may occur after surgery. Therefore, reconstruction is recommended in chronic injuries. Also, the alignment of lower extremity and gait patterns are considerably important in chronic cases. Diagnosis and treatment of limb mal-alignment should not be overlooked in the management of chronic ligamentous instability ${ }^{15)}$. If there is more than $3^{\circ}$ of varus deviation or the hip-knee axis passes within $30 \%$ of the medial side of the tibial plateau, high tibial osteotomy can be considered.

Reconstruction of the injured PLC structures can be performed anatomically and non-anatomically. Anatomic reconstruction of the injured PLC structures is more recommendable than non-anatomic reconstruction because non-anatomic and non-isometric reconstruction can increase the risk of limitation in knee joint movement, eventually leading to treatment failure.

PCL and PLC injuries are not common knee injuries and are likely to be overlooked in the diagnostic process. In addition, sufficient data on treatment results are not available compared to other common knee injuries. Therefore, there is a need for con- tinuous accumulation of various research results.

\section{References}

1. Fanelli GC, Edson CJ. Posterior cruciate ligament injuries in trauma patients: part II. Arthroscopy. 1995;11:526-9.

2. Amis AA, Gupte CM, Bull AM, Edwards A. Anatomy of the posterior cruciate ligament and the meniscofemoral ligaments. Knee Surg Sports Traumatol Arthrosc. 2006;14:25763.

3. Mauro CS, Sekiya JK, Stabile KJ, Haemmerle MJ, Harner CD. Double-bundle PCL and posterolateral corner reconstruction components are codominant. Clin Orthop Relat Res. 2008;466:2247-54.

4. Baker CL Jr, Norwood LA, Hughston JC. Acute combined posterior cruciate and posterolateral instability of the knee. Am J Sports Med. 1984;12:204-8.

5. Covey DC, Sapega AA, Sherman GM. Testing for isometry during reconstruction of the posterior cruciate ligament: anatomic and biomechanical considerations. Am J Sports Med. 1996;24:740-6.

6. Harner CD, Janaushek MA, Kanamori A, Yagi M, Vogrin TM, Woo SL. Biomechanical analysis of a double-bundle posterior cruciate ligament reconstruction. Am J Sports Med. 2000;28:144-51.

7. Mannor DA, Shearn JT, Grood ES, Noyes FR, Levy MS. Two-bundle posterior cruciate ligament reconstruction: an in vitro analysis of graft placement and tension. Am J Sports Med. 2000;28:833-45.

8. Race A, Amis AA. PCL reconstruction: in vitro biomechanical comparison of 'isometric' versus single and doublebundled 'anatomic' grafts. J Bone Joint Surg Br. 1998;80:1739.

9. Krukhaug Y, Molster A, Rodt A, Strand T. Lateral ligament injuries of the knee. Knee Surg Sports Traumatol Arthrosc. 1998;6:21-5.

10. Kannus P. Nonoperative treatment of grade II and III sprains of the lateral ligament compartment of the knee. Am J Sports Med. 1989;17:83-8.

11. Geeslin AG, LaPrade RF. Outcomes of treatment of acute grade-III isolated and combined posterolateral knee injuries: a prospective case series and surgical technique. J Bone Joint Surg Am. 2011;93:1672-83.

12. Levy BA, Dajani KA, Morgan JA, Shah JP, Dahm DL, Stuart MJ. Repair versus reconstruction of the fibular collateral ligament and posterolateral corner in the multiligament- 
Knee Surg Relat Res, Vol. 29, No. 4, Dec. 2017245

injured knee. Am J Sports Med. 2010;38:804-9.

13. Shelbourne KD, Haro MS, Gray T. Knee dislocation with lateral side injury: results of an en masse surgical repair technique of the lateral side. Am J Sports Med. 2007;35:1105-16.

14. Spitzer E, Doyle JB, Marx RG. Outcomes of surgical treatment of posterolateral instability of the knee. J Knee Surg.
2015;28:471-4.

15. Savarese E, Bisicchia S, Romeo R, Amendola A. Role of high tibial osteotomy in chronic injuries of posterior cruciate ligament and posterolateral corner. J Orthop Traumatol. 2011; 12:1-17. 